\title{
ARTICLE
}

Clinical Study

\section{Adding cetuximab to paclitaxel and carboplatin for first-line treatment of carcinoma of unknown primary (CUP): results of the Phase 2 AIO trial PACET-CUP}

Gunnar Folprecht ${ }^{1}$, Karolin Trautmann ${ }^{1}$, Alexander Stein ${ }^{2}$, Gerdt Huebner ${ }^{3}$, Michael Stahl ${ }^{4}$, Stefan Kasper ${ }^{5}$, Albrecht Kretzschmar ${ }^{6}$, Claus-Henning Köhne ${ }^{7}$, Viktor Grünwald $\mathbb{B}^{8,9}$, Ralf-Dieter Hofheinz ${ }^{10}$, Katharina Schütte ${ }^{1}$, Harald Löffler ${ }^{11}$, Carsten Bokemeyer ${ }^{2}$, Alwin Krämer ${ }^{12}$ and Arbeitsgemeinschaft Internistische Onkologie (AIO) - CUP Group

BACKGROUND: Patients with carcinoma of unknown primary (CUP) have a dismal prognosis, even when treated with multi-agent chemotherapy. We hypothesised that adding the epidermal growth-factor receptor (EGFR) inhibitor cetuximab to standard first-line chemotherapy with paclitaxel and carboplatin would improve PFS and RR in unfavourable CUP.

METHODS: This open-labelled, multicentre Phase 2 study included patients with unfavourable, untreated adeno- or undifferentiated CUP. Patients were randomised to receive either paclitaxel/carboplatin (group A) or paclitaxel/carboplatin plus cetuximab (group B) every 3 weeks for a maximum of 6 cycles followed by cetuximab maintenance in group B. The primary endpoint was PFS in the two groups. Secondary endpoints were RR, toxicity and overall survival (OS).

RESULTS: One-hundred-and-fifty patients were randomised (group $A=72$, group $B=78$ ). The median PFS and OS for all patients were 3.8 and 8.1 months (95\% confidence interval $(\mathrm{Cl}): 2.9-4.8$ and 6.8-9.5). There was no significant difference in PFS (3.7 vs 4.6 months, HR 0.98) or OS ( 8.1 vs 7.4, HR 1.1) between the two treatment groups. Response rate tended to be better for chemotherapy plus cetuximab compared to chemotherapy alone ( $22 \%$ vs $15 \%)$. Adverse events grade $\geq 3$ were comparable between the two groups, except for significantly increased skin toxicity in the cetuximab arm.

CONCLUSIONS: Cetuximab plus paclitaxel/carboplatin did not improve PFS, OS and RR in metastatic CUP compared to paclitaxel/ carboplatin alone. Addition of cetuximab resulted in additional skin toxicity.

CLINICAL TRIAL REGISTRATION: The study was registered at clinicaltrials.gov as NCT00894569.

British Journal of Cancer (2021) 124:721-727; https://doi.org/10.1038/s41416-020-01141-8

\section{BACKGROUND}

Carcinoma of unknown primary (CUP) is a challenging oncological problem defined as a metastatic cancer without a clinically evident primary tumour. It accounts for approximately $3 \%$ of all newly diagnosed advanced cancers with declining incidence during recent years. ${ }^{1}$ Patients with CUP have a dismal prognosis with a median overall survival of less than 1 year. $^{2}$ The poor prognosis of CUP probably results from the aggressive nature of the disease ${ }^{3}$ and from a commonly encountered delay in specific diagnosis. ${ }^{4}$

During recent years, there has been substantial progress in more accurately defining the tissue of origin of CUP tissue biopsies using gene expression profiling and more advanced immunohistochemistry. ${ }^{5,6}$ However, most patients with CUP still receive empiric chemotherapy. A commonly accepted first-line regimen is a platinum/taxane combination that yields response rates (RR) of around $20-30 \%$ with a median overall survival (OS) of 9-10 months in selected patients. $^{7-9}$ Several clinical trials have aimed to improve treatment results by combining standard chemotherapy with novel, molecular targeted therapies. ${ }^{10-12}$

Cetuximab is an anti-epidermal growth-factor receptor (EGFR) monoclonal antibody approved for the treatment of squamous cell head and neck and RAS wild-type colorectal cancer. In combination with standard first-line chemotherapy regimens, it significantly improves clinical outcome in the treatment of metastatic squamous cell carcinoma of the head and neck ${ }^{13}$ and metastatic colorectal cancer. ${ }^{14,15}$ Besides, inhibition of EGFR with the small-molecule erlotinib has been registered for the treatment of pancreatic cancer. $^{16}$ Occult lung and pancreatic cancers are the most commonly identified primary tumours at autopsy in patients with

\footnotetext{
${ }^{1}$ Technische Universität Dresden/University Hospital Carl Gustav Carus, Medical Department I, Dresden, Germany; ${ }^{2}$ University Medical Center Hamburg-Eppendorf, Hamburg, Germany; ${ }^{3}$ oho! ostholstein-onkologie, Oldenburg i.H., Germany; ${ }^{4}$ Evangl. Kliniken Essen-Mitte, Department of Medical Oncology, Essen, Germany; ${ }^{5}$ West German Cancer Centre, University Hospital Essen, Department of Medical Oncology, Essen, Germany; ${ }^{6}$ MVZ Mitte, Leipzig, Germany; ${ }^{7}$ University Clinic for Internal Medicine, Oncology und Hematology, Oldenburg, Germany; ${ }^{8}$ Department of Hematology, Hemostaseology, Oncology \& Stem Cell Transplantation, Hannover Medical School, Hannover, Germany; ${ }^{9}$ West-German Cancer Centre Essen, University Hospital Essen, Essen, Germany; ${ }^{10}$ University Medical Center Mannheim, Tagestherapiezentrum am ITM, Mannheim, Germany; ${ }^{11}$ Marienhospital,

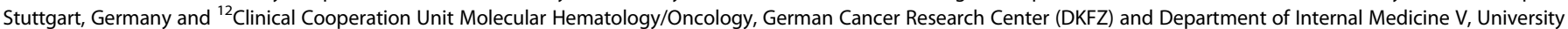
of Heidelberg, Heidelberg, Germany

Correspondence: Gunnar Folprecht (gunnar.folprecht@uniklinikum-dresden.de)
}

Received: 25 May 2020 Revised: 1 October 2020 Accepted: 15 October 2020

Published online: 25 November 2020 
CUP. ${ }^{17}$ Therefore, we hypothesised that the inhibition of EGFR by adding cetuximab to standard first-line chemotherapy would improve RR and progression-free survival (PFS) in patients with CUP.

Here we report the results of the PACET-CUP study, a randomised, multicentre Phase 2 study evaluating the efficacy and safety of adding cetuximab to paclitaxel/carboplatin for firstline treatment of adeno- and undifferentiated CUP.

\section{METHODS}

\section{Patient eligibility}

Patients with adeno- or undifferentiated CUP were eligible. CUP histology had to be confirmed by the local pathologist. Tumours displaying any immunohistochemistry pattern indicative for a specific entity (i.e. Her-2 positive or hormone-receptor positive corresponding to breast cancer or CK7-negative/CK20-positive suggestive of colorectal cancer, carcinomas with neuroendocrine differentiation) were excluded. The study protocol intended central pathological review. However, central analysis could only be performed in 20 patients. For all other cases, tumour material was not sufficient for central review.

Eligible patients had to meet the following criteria: WHO performance status $0-1$, measurable tumour lesion according to RECIST, age $>/=18$ years, adequate liver and renal function (defined as bilirubin $\leq 1.5 \times$ upper-normal level (UNL), ASAT and ALAT $\leq 2.5 \times$ UNL or in the case of liver metastases $\leq 5 \mathrm{UNL}$, serum creatinine $\leq 1.5$ $\mathrm{UNL}$ ) and adequate bone marrow function (defined as neutrophil count $\geq 1.5 \times 10^{9} / \mathrm{L}$, platelet count $\geq 100 \times 10^{9} / \mathrm{L}$ and haemoglobin $>5 \mathrm{mmol} / \mathrm{l})$. Specific patient subsets with clinically favourable CUP as described in current guidelines ${ }^{18}$ were excluded: females with axillary-node metastasis or peritoneal carcinomatosis, as well as younger males ( $<50$ years) with retroperitoneal or mediastinal lymph nodes as the predominant tumour site. A standard diagnostic approach was applied to rule out a definite primary tumour site. This approach consisted of physical examination, tumour marker screening (CEA, CA 19-9 and AFP; for female patients: CA 15-3 and CA 125; for male patients: PSA and B-HCG), an oesophagogastroduodenoscopy and abdominal-pelvic and thoracic, contrast-enhanced spiral $C T$, MRI or PET examination. Female patients received a gynaecologic examination and mammography. All patients provided written informed consent.

\section{Study design}

This multicentre, open-labelled, randomised Phase 2 study (registered at clinicaltrials.gov as NCT00894569) was performed at 13 sites throughout Germany. The study protocol was approved by local ethics committees of the participating centres and the competent authorities. Eligible patients were centrally randomised $(1: 1)$ to receive paclitaxel/carboplatin (group A) or paclitaxel/ carboplatin plus cetuximab (group B) and stratified by age, sex, presence of liver metastases and LDH level (normal vs above normal). Patients in group A received paclitaxel $175 \mathrm{mg} / \mathrm{m}^{2}$ plus carboplatin at an area under the concentration time curve (AUC) of 5 . Chemotherapy was repeated every 21 days for a maximum of 6 cycles. Therapy in group B consisted of the same paclitaxel/ carboplatin regimen preceded by cetuximab. The first cetuximab infusion contained $400 \mathrm{mg} / \mathrm{m}^{2}$ followed by weekly doses of $250 \mathrm{mg} / \mathrm{m}^{2}$. After 6 cycles of chemotherapy, patients in group B received maintenance therapy with weekly cetuximab. Completion of all six cycles of therapy was not a mandatory prerequisite to start maintenance. Chemotherapy and cetuximab dose modifications and treatment alterations were done according to the manufacturer's recommendation. Therapy continued until progression, unacceptable toxicity or withdrawal of consent. During the study, tumour evaluation according to Response Evaluation Criteria in Solid Tumours (RECIST 1.0) ${ }^{19}$ using CT or MRI had to be performed by the local investigator/radiologist every 8 weeks.
Study objectives

The primary endpoint of the study was PFS rate at week 32 ( 8 months) after randomisation. The PFS rate was defined as the proportion of patients alive with stable disease (SD), partial response (PR) or complete response (CR) according to RECIST (version 1.0). ${ }^{19}$ Secondary endpoints included median PFS, overall RR (proportion of patients with a CR or PR as their best measured response) and toxicity in the two treatment groups (according to $\mathrm{NCl}-\mathrm{CTC}$ version 3.0), as well as OS time defined as the time from randomisation to death in the intention-to-treat (ITT) population.

\section{Statistical plan}

Based on the results of a previous study by the AIO CUP working group, ${ }^{9}$ we assumed a PFS rate at 32 weeks of $24 \%$ with standard treatment and $46 \%$ with the cetuximab-combination therapy. With a scheduled interim analysis after 50 patients, a one-sided alpha error of 0.05 and a power of $85 \%, 150$ patients were planned to be randomised.

We used the Kaplan-Meier method to analyse PFS and OS and to estimate their medians. Survival rates between the two treatment arms were compared using the log-rank and chisquare test. Logistic regression and Cox regression analyses were performed to describe treatment effects in the different subgroups. We conducted multivariate analysis stratified by treatment arm to determine the influence of any baseline parameter on PFS and OS. Multivariate analysis was repeated using both treatment arms as variables.

\section{RESULTS}

Study population

Between March 15, 2010 and March 20, 2017, one-hundred-andfifty patients were randomised to paclitaxel/carboplatin (group $A$, $n=72$ ) or paclitaxel/carboplatin/cetuximab (group $\mathrm{B}, n=78$ ). Patient characteristics are summarised in Table 1.

\begin{tabular}{|c|c|c|c|}
\hline & $\begin{array}{l}\text { Arm A } \\
\text { (Chemotherapy) }\end{array}$ & $\begin{array}{l}\text { Arm B } \\
\text { (Chemotherapy } \\
\text { + cetuximab) }\end{array}$ & All patients \\
\hline & $N=72$ & $N=78$ & $N=150$ \\
\hline Age (median) & $59 y$. & $62.5 \mathrm{y}$. & $61 \mathrm{y}$. \\
\hline Age $>65 \mathrm{y}$. & $26(36 \%)$ & $33(42 \%)$ & $59(39 \%)$ \\
\hline Gender: male & 32 (44\%) & $34(44 \%)$ & $66(44 \%)$ \\
\hline WHO PS 0 & 24 (33\%) & $34(45 \%)$ & $58(39 \%)$ \\
\hline WHO PS 1 & 47 (65\%) & $42(55 \%)$ & 89 (60\%) \\
\hline WHO PS 2 & $1(1 \%)$ & $0(0 \%)$ & $1(1 \%)$ \\
\hline Leucocytes > 10 & $29(40 \%)$ & $25(32 \%)$ & $54(36 \%)$ \\
\hline $\mathrm{LDH}>\mathrm{ULN}$ & $46(64 \%)$ & $53(68 \%)$ & $99(66 \%)$ \\
\hline Liver met. & $38(53 \%)$ & $34(44 \%)$ & $72(48 \%)$ \\
\hline Lung met. & 16 (22\%) & $20(29 \%)$ & $36(28 \%)$ \\
\hline Lymph-node met. & 37 (51\%) & $41(53 \%)$ & $78(52 \%)$ \\
\hline Bone met. & $6(8 \%)$ & $7(9 \%)$ & $13(10 \%)$ \\
\hline \multicolumn{4}{|l|}{ No. of met. sites* } \\
\hline 1 site & 16 (22\%) & $11(14 \%)$ & $27(18 \%)$ \\
\hline 2 sites & 25 (35\%) & 24 (31\%) & 49 (33\%) \\
\hline 3 sites & $17(24 \%)$ & $26(33 \%)$ & $43(29 \%)$ \\
\hline 4 sites & $8(11 \%)$ & $11(14 \%)$ & $19(13 \%)$ \\
\hline 5 sites & $4(6 \%)$ & $3(4 \%)$ & $7(5 \%)$ \\
\hline 6 sites & $2(3 \%)$ & $3(4 \%)$ & $5(3 \%)$ \\
\hline
\end{tabular}

*One metastatic site corresponds to one involved organ system (i.e. liver, lung...). 
The median age of the whole study population was 61 years with slightly more women included (56\%). All but one patient had WHO performance status 0 or 1 . The number of involved organ sites, the presence of liver, lung, bone or lymph-node metastases

\begin{tabular}{|c|c|c|c|c|}
\hline \multirow{3}{*}{$\begin{array}{l}\text { Adverse events } \\
\text { Grade } \geq 3 \\
\text { Leukopenia/neutropenia }\end{array}$} & \multirow{2}{*}{\multicolumn{2}{|c|}{$\begin{array}{l}\begin{array}{l}\text { Arm A } \\
\text { (chemo) }\end{array} \\
N=72\end{array}$}} & \multirow{2}{*}{\multicolumn{2}{|c|}{$\begin{array}{l}\begin{array}{l}\text { Arm B } \\
\text { (chemo }+ \\
\text { cetux) }\end{array} \\
N=78\end{array}$}} \\
\hline & & & & \\
\hline & 14 & $(19 \%)$ & 23 & $(29 \%)$ \\
\hline Febrile neutropenia & 0 & $(0 \%)$ & 2 & $(3 \%)$ \\
\hline Thrombopenia & 2 & $(3 \%)$ & 4 & $(5 \%)$ \\
\hline Anaemia & 4 & $(6 \%)$ & 5 & $(6 \%)$ \\
\hline Skin toxicity & 0 & $(0 \%)$ & 14 & $(18 \%)$ \\
\hline Mucositis & 2 & $(3 \%)$ & 2 & $(3 \%)$ \\
\hline Diarrhoea & 5 & $(7 \%)$ & 5 & $(6 \%)$ \\
\hline Nausea/vomiting & 2 & $(3 \%)$ & 2 & $(3 \%)$ \\
\hline Increased liver enzymes & 2 & (3\%) & 1 & $(1 \%)$ \\
\hline Other gastrointestinal events & 3 & $(4 \%)$ & 6 & $(8 \%)$ \\
\hline Fatigue/decreased performance status & 4 & $(6 \%)$ & 7 & (9\%) \\
\hline Thromboembolic events & 4 & $(6 \%)$ & 4 & $(5 \%)$ \\
\hline Syncope/falls & 0 & $(0 \%)$ & 2 & $(3 \%)$ \\
\hline Arrhythmia & 0 & $(0 \%)$ & 2 & $(3 \%)$ \\
\hline Other cardiovascular events & 5 & (7\%) & 6 & $(8 \%)$ \\
\hline Polyneuropathy & 3 & $(4 \%)$ & 4 & $(5 \%)$ \\
\hline Hypersensitivity & 2 & $(3 \%)$ & 2 & $(3 \%)$ \\
\hline Pain & 12 & $(17 \%)$ & 13 & $(17 \%)$ \\
\hline Other neurological events & 2 & (3\%) & 2 & $(3 \%)$ \\
\hline Hypomagnesaemia & 0 & $(0 \%)$ & 4 & $(5 \%)$ \\
\hline Renal events & 2 & (3\%) & 0 & $(0 \%)$ \\
\hline Other lab events & 3 & $(4 \%)$ & 2 & $(3 \%)$ \\
\hline Ascites/pleural effusion & 3 & $(4 \%)$ & 2 & $(3 \%)$ \\
\hline Infections & 3 & $(4 \%)$ & 5 & $(6 \%)$ \\
\hline Other events & 4 & $(6 \%)$ & 2 & (3\%) \\
\hline
\end{tabular}

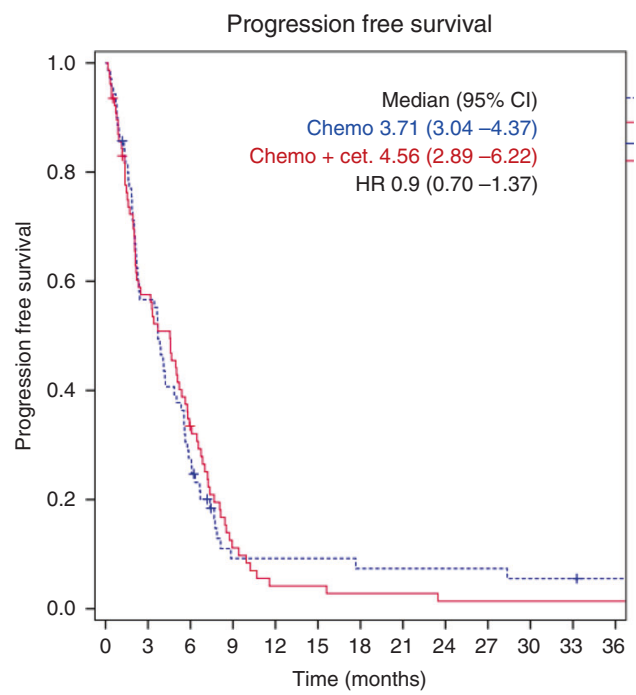

was well balanced between the two treatment groups. Similarly, the percentage of elevated LDH or leucocyte counts was equally distributed. There was no significant correlation between the number of metastatic sites and elevated LDH, increased leucocytes or WHO performance status.

Treatment duration and toxicity

The whole study population received a median of three cycles of chemotherapy [quartile range 2.0-6.0]. Twenty-seven patients in arm A (37\%) and 24 patients in arm B (31\%) completed all six cycles. Thirty patients in arm B (38\%) started with cetuximabmaintenance therapy. In 6 patients, carboplatin and paclitaxel had to be stopped due to toxicity, and maintenance was started before completing all 6 cycles of chemotherapy. All treatment-related adverse events $>/=$ grade 3 for the 2 study groups are summarised in Table 2. The most common higher-grade adverse event

\begin{tabular}{|c|c|c|c|}
\hline & $\begin{array}{l}\text { Arm A } \\
\text { (Chemo) }\end{array}$ & $\begin{array}{l}\text { Arm B } \\
\text { (Chemo + Cet) }\end{array}$ & All patients \\
\hline & $N=72$ & $N=78$ & $N=150$ \\
\hline \multicolumn{4}{|c|}{ Tumour response } \\
\hline \multirow[t]{2}{*}{ PR } & 11 & 17 & 28 \\
\hline & $15 \%$ & $22 \%$ & $19 \%$ \\
\hline 95\% Cl: & $7.9-26 \%$ & $13-33 \%$ & $13-26 \%$ \\
\hline SD & 25 & 25 & 50 \\
\hline PD & 31 & 32 & 63 \\
\hline NA & 5 & 4 & 9 \\
\hline \multicolumn{4}{|c|}{ Progression-free survival } \\
\hline Median & 3.71 & 4.56 & 3.84 \\
\hline $95 \% \mathrm{Cl}$ & $3.04-4.37$ & $2.89-6.22$ & $2.90-4.77$ \\
\hline HR & 0.98 & $95 \% \mathrm{Cl}: 0.70-1.37$ & \\
\hline \multicolumn{4}{|c|}{ Overall survival } \\
\hline Median & 8.13 & 7.38 & 8.13 \\
\hline $95 \% \mathrm{Cl}$ & $6.46-9.80$ & $5.12-9.64$ & $6.81-9.46$ \\
\hline$H R$ & 1.10 & $95 \%$ Cl: 0.77-1.56 & \\
\hline
\end{tabular}

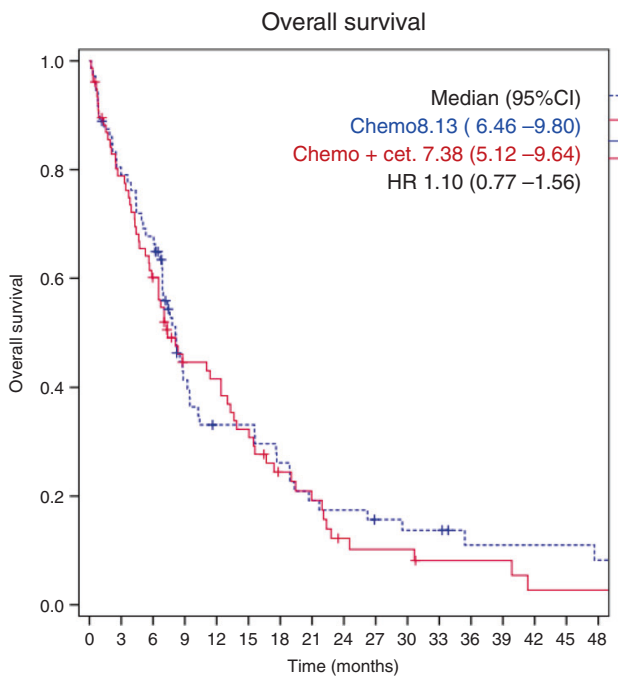

Fig. 1 Progression-free and overall survival according to treatment arm. The graphs show the progression-free survival and overall survival probability for patients in arms A (blue dotted line) and B (red solid lines). 
a

Patients with complete or partial remission

$\begin{array}{lcc} & \text { All patients } \\ & n / N & \\ \text { Age }>65 \text { y. } & 10 / 59 & (17 \%) \\ \text { Age } \leq 65 y . & 18 / 91 & (20 \%) \\ \text { Gender: male } & 14 / 84 & (17 \%) \\ \text { Gender: female } & 14 / 66 & (21 \%) \\ \text { WHO PS 0 } & 13 / 60 & (22 \%) \\ \text { WHO PS 1-2 } & 15 / 90 & (17 \%) \\ \text { Leukocytes }>10 & 9 / 54 & (17 \%) \\ \text { Leukocytes } \leq 10 & 19 / 96 & (20 \%) \\ \text { LDH }>\text { ULN } & 20 / 99 & (20 \%) \\ \text { LDH } \leq \text { ULN } & 8 / 51 & (16 \%) \\ \text { Liver met. } & 15 / 72 & (21 \%) \\ \text { No known liver met. } & 13 / 78 & (17 \%) \\ \text { Lung met. } & 8 / 36 & (22 \%) \\ \text { No known lung met. } & 20 / 114 & (18 \%) \\ \text { Lymph node met. } & 18 / 78 & (23 \%) \\ \text { No known LN met } & 10 / 72 & (14 \%) \\ \text { Bone met. } & 1 / 13 & (8 \%) \\ \text { No known bone met. } & 27 / 137 & (20 \%) \\ >\text { 2 met sites } & 16 / 74 & (22 \%) \\ \leq 2 \text { met sites } & 12 / 76 & (16 \%) \\ \text { All pts } & \mathbf{2 8 / 1 5 0} & (\mathbf{2 0 \% )}\end{array}$

$\begin{array}{ccc}\begin{array}{c}\text { Arm A (chemo) } \\ \text { n/N }\end{array} & \text { Arm B (chemo } \\ \mathbf{n} / \boldsymbol{N} \\ 4 / 25 & (16 \%) & 6 / 34 \\ 7 / 47 & (15 \%) & 11 / 44 \\ 7 / 40 & (18 \%) & 7 / 44 \\ 4 / 32 & (13 \%) & 10 / 34 \\ 3 / 24 & (13 \%) & 10 / 36 \\ 8 / 48 & (17 \%) & 7 / 42 \\ 5 / 30 & (17 \%) & 4 / 24 \\ 6 / 42 & (14 \%) & 13 / 54 \\ 8 / 46 & (17 \%) & 12 / 53 \\ 3 / 26 & (12 \%) & 5 / 25 \\ 3 / 39 & (8 \%) & 12 / 33 \\ 8 / 33 & (24 \%) & 5 / 45 \\ 2 / 16 & (13 \%) & 6 / 20 \\ 9 / 56 & (16 \%) & 11 / 58 \\ 7 / 38 & (18 \%) & 11 / 40 \\ 4 / 34 & (12 \%) & 6 / 38 \\ 0 / 6 & (0 \%) & 1 / 7 \\ 11 / 66 & (17 \%) & 16 / 71 \\ 4 / 31 & (13 \%) & 12 / 43 \\ 7 / 41 & (17 \%) & 5 / 35 \\ 11 / 72 & (17 \%) & 17 / 78\end{array}$

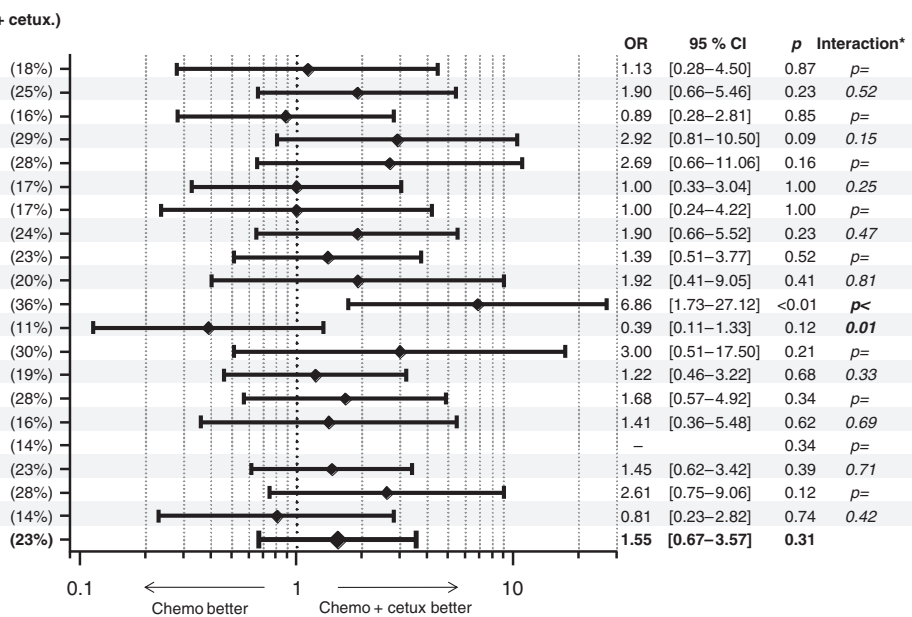

b

Progression-free survival

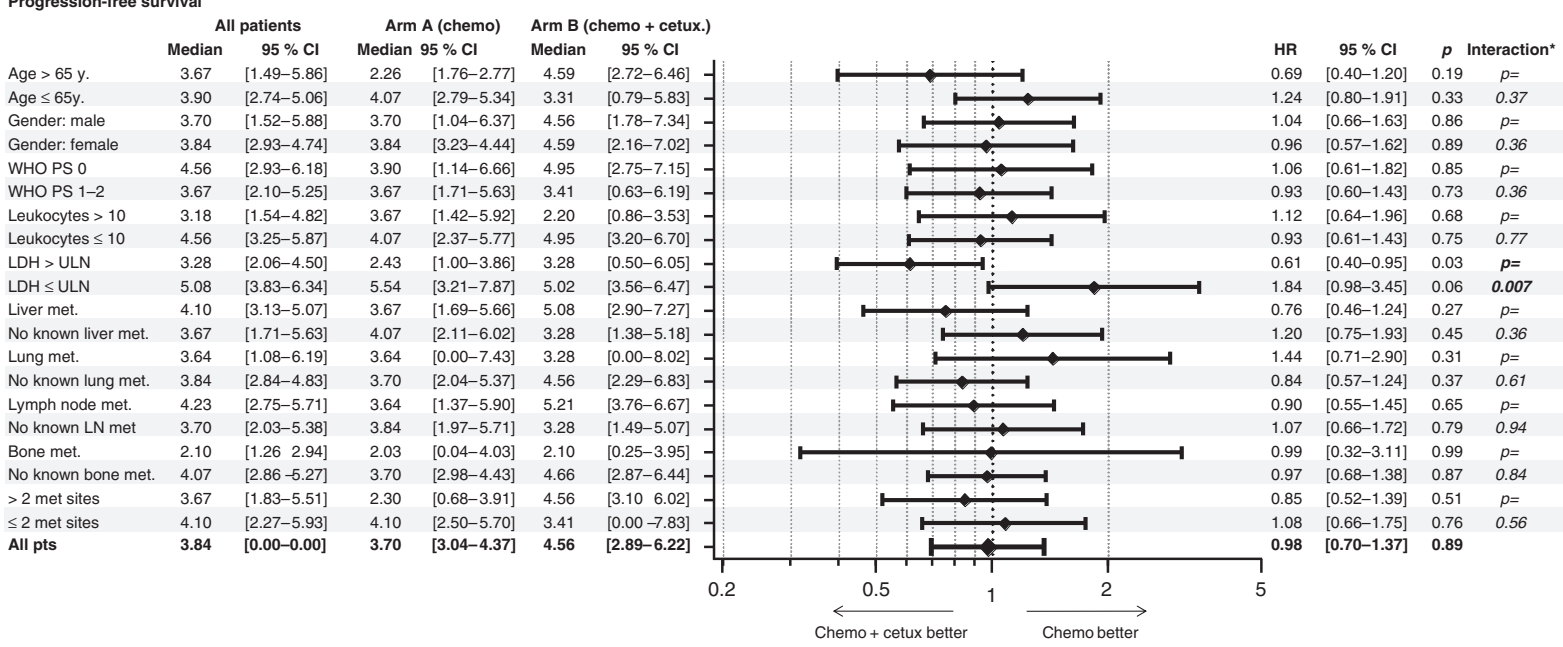

C

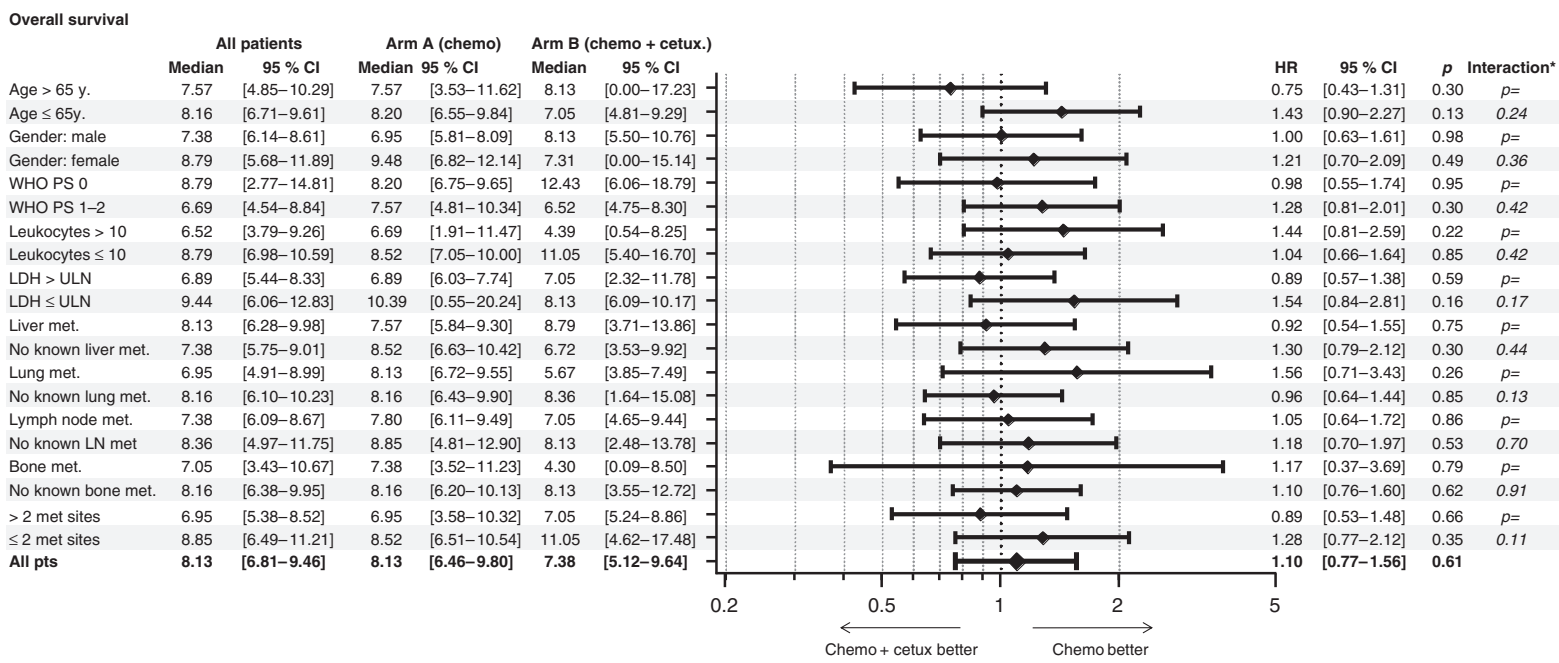

Fig. 2 Treatment efficacy according to subgroups. The Forrest plots describe the Odd's ratio for patients with complete or partial remission (a) and the Hazard ratio for progression free survival (b) or overall survival (c) according to the baseline parameters (univariat analysis). * interaction between baseline parameter and treatment arm.

in both treatment groups was leukopenia, which tended to occur more often in the cetuximab group. Skin toxicity was the only adverse event $>/=$ grade 3 that was significantly increased in the experimental arm ( $0 \%$ in arm A vs $18 \%$ in arm $B)$.
Treatment efficacy

The PFS at week 32-which represented the primary endpointwas $19.5 \%$ [95\% Cl: $10.5-28.5 \%$ ] in patients receiving chemotherapy plus cetuximab (arm B) compared to $12.9 \%[95 \% \mathrm{Cl}$ : 
Table 4. Multivariate analysis for progression free survival and overall survival.

\begin{tabular}{|c|c|c|c|c|c|c|}
\hline & \multicolumn{3}{|c|}{ Progression-free survival } & \multicolumn{3}{|c|}{ Overall survival } \\
\hline Lung met. & 1.22 & {$[0.80-1.87]$} & $p=0.35$ & 1.38 & {$[0.88-2.18]$} & $p=0.17$ \\
\hline Lymph-node met. & 0.99 & {$[0.69-1.42]$} & $p=0.97$ & 1.25 & {$[0.86-1.81]$} & $p=0.25$ \\
\hline Bone met. & 1.62 & {$[0.88-2.99]$} & $p=0.12$ & 1.69 & {$[0.92-3.08]$} & $p=0.09$ \\
\hline Liver met. & 0.93 & {$[0.63-1.36]$} & $p=0.69$ & 1.07 & {$[0.71-1.61]$} & $p=0.74$ \\
\hline Age $>65$ & 1.10 & {$[0.75-1.60]$} & $p=0.64$ & 1.00 & {$[0.68-1.47]$} & $p=0.99$ \\
\hline WHO PS 1-2 & 1.22 & {$[0.85-1.74]$} & $p=0.29$ & 1.67 & {$[1.13-2.46]$} & $p=0.01$ \\
\hline Gender (male) & 1.14 & {$[0.78-1.66]$} & $p=0.49$ & 1.19 & {$[0.81-1.74]$} & $p=0.38$ \\
\hline No of met sites $>2$ & 1.14 & {$[0.78-1.66]$} & $p=0.49$ & 1.12 & {$[0.76-1.66]$} & $p=0.58$ \\
\hline
\end{tabular}

Table 5. Trials in patients with CUP treatment with carboplatin/paclitaxel combinations.

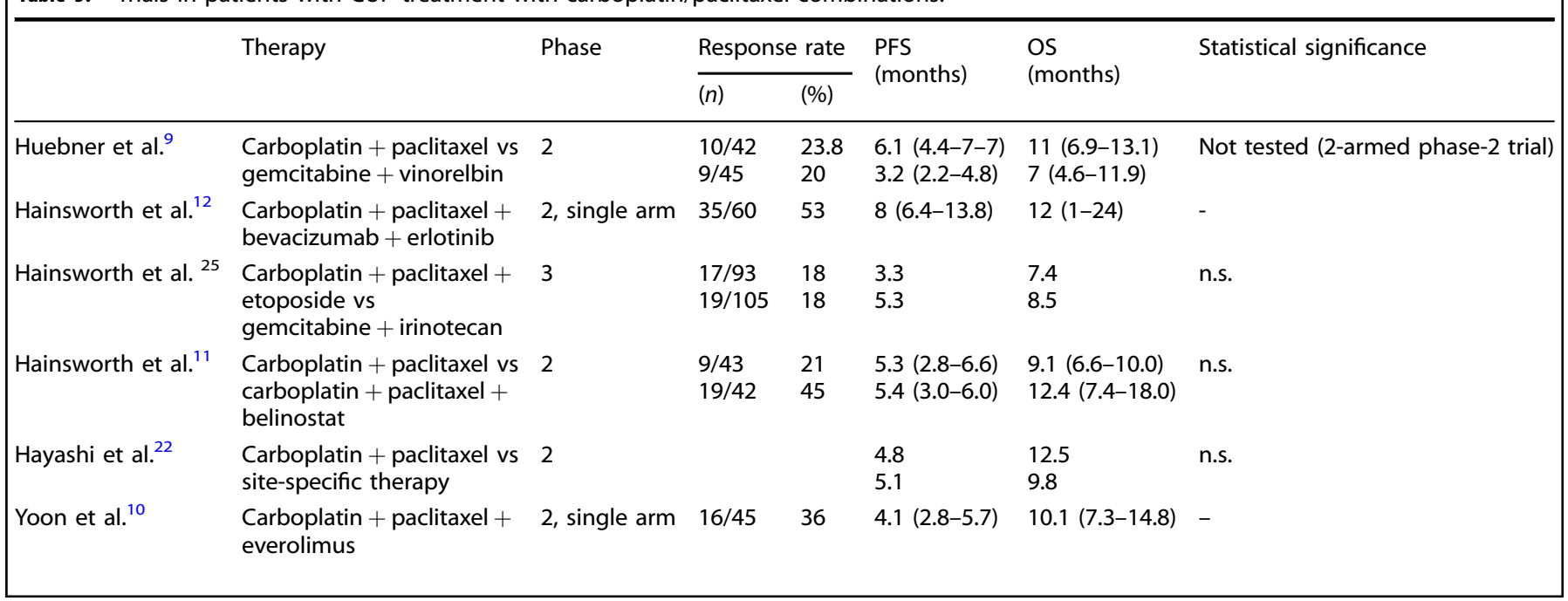

4.5-21.3\%] in patients receiving chemotherapy alone (arm A). This difference did not reach statistical significance. Similarly, the median PFS and OS were not significantly different between the two treatment arms (Table 3 and Fig. 1). The tumour-response rate in arm B was $22 \%$ compared to $15 \%$ in arm A, which was not significantly different.

\section{Subgroup analysis}

The subgroup analysis according to baseline characteristics demonstrated a higher response to chemotherapy plus cetuximab than to chemotherapy alone in patients with liver metastases (odds ratio 6.86 [95\% Cl: 1.73-27.12]) and a longer PFS with cetuximab in patients with elevated LDH (hazard ratio 0.61 [95\% $\mathrm{Cl}$ : 0.40-0.95]). There was no significant interaction between baseline parameters and treatment group with regard to overall survival for any subgroup analysed (Fig. 2).

A multivariate analysis stratified by treatment arm was performed to determine the influence of baseline characteristics on PFS, OS and RR. Elevated leucocyte count and impaired performance status were significantly associated with poorer OS. An elevated LDH correlated with a shorter PFS (Table 4). Adding the treatment arm as a variable did not change the results.

\section{DISCUSSION}

To our knowledge, this is the second largest clinical trial prospectively comparing the addition of a targeted agent to standard chemotherapy in patients with unfavourable CUP. There was no clinically relevant benefit from the combination of cetuximab and paclitaxel/carboplatin compared to paclitaxel/ carboplatin alone despite numerically, but not statistically significant higher response and PFS rates at 8 months.

A smaller, non-randomised trial, including 60 patients with CUP, previously evaluated concurrent EGFR and vascular endothelial growth-factor (VEGF) blockage by adding both, erlotinib and bevacizumab to chemotherapy with paclitaxel/carboplatin. ${ }^{12}$ In agreement with our results, therapy was well tolerated with no new or unexpected side effects identified. The authors described an overall RR of $53 \%$ and a PFS of $38 \%$ at 1 year in their patient population. Another, more recent trial found that the addition of everolimus to carboplatin and paclitaxel resulted in a RR of $36 \%$ and a median PFS of 4.1 months. ${ }^{10}$ Of note, both studies were non-randomised. Another randomised Phase 2 trial showed that the addition of belinostat, a histone deacetylase inhibitor to paclitaxel/carboplatin, did not improve PFS of patients with CUP who were receiving first-line therapy. ${ }^{11}$ 
The median PFS of 3.8 months observed across treatment groups in our study is in agreement with other published data. Table 5 summarises available results from the most important clinical trials evaluating carboplatin/paclitaxel plus/minus experimental agents for the treatment of CUP. Since our trial included 150 patients from 13 different centres throughout Germany, we believe that our results are a good reflection of real-world data.

As in other types of cancer, specific mutations may increase sensitivity or result in resistance to anti-EGFR therapy. For example, in metastatic colorectal cancer, only tumours without mutations in KRAS and NRAS respond to therapy with anti-EGFR antibodies. $^{20}$ It is possible that specific molecular CUP subsets might benefit from the addition of EGFR inhibition. However, due to insufficient tumour material in the majority of cases, we were not able to perform molecular analyses and collect this information. Techniques using circulating tumour DNA were not yet available when our study was conducted.

Since 2009, when the current study was designed, diagnostic approaches to classify CUP for clinical studies have changed substantially. Molecular tumour profiling helps to accurately predict the tissue of origin in many cases of CUP and might aid to select tumour site-specific therapies. ${ }^{5,6,10,21}$ However, in a randomised Phase 2 trial using gene expression profiling to enable site-specific treatment for patients with CUP, this approach did not result in a significant improvement of PFS or OS compared with empirical chemotherapy. ${ }^{22}$ Similarly, the results from a recently presented European Phase 3 trial including 243 patients did not show superior outcomes for patients with CUP treated with therapy tailored to the suspected primary site of origin as identified by molecular analysis. ${ }^{23}$ These studies are in line with a current meta-analysis that could not find a survival benefit for site-specific treatments in CUP. ${ }^{24}$

Adding cetuximab to standard chemotherapy failed to improve the clinical outcome of patients with CUP. With a median OS of 8.1 months across all patients enrolled, our trial confirms the poor prognosis of this disease and underlines the medical need for better treatment options. Clinical trials enabling early biomarkerdriven targeted therapies by using more advanced molecular and immune-profiling techniques are underway.

\section{ACKNOWLEDGEMENTS}

We thank all patients and their relatives as well as all investigators and study nurses for the great support of this academic trial.

\section{AUTHOR CONTRIBUTIONS}

G.F., A.K.R.Ä. and K.S. conceived and designed the trial and wrote the study protocol. G.F., K.T., A.S., G.H., M.S., S.K., A.K.R.E., C.H.K., V.G., R.H., H.L., C.B. and A.K.R.Ä. enrolled patients into the study and were responsible for patient management and data collection at their site. G.F. conducted statistical data analysis. G.F., K.T., G.H. and A.K.R. Ä. analysed the data. K.T. and G.F. wrote the paper. All authors read and approved the final paper prior to submission.

\section{ADDITIONAL INFORMATION}

Ethics approval and consent to participate The legal sponsor of the study was the University of Heidelberg, Germany. The study protocol was approved by the ethics committee of the University of Heidelberg, Germany (leading ethics committee in Germany for this trial, reference number AFmu-408/2008) and by the responsible local ethics committees of all participating centres (ethics committees of the Technische Universität Dresden, Medizinische Hochschule Hannover, Medizinische Fakultät für Klinische Medizin Mannheim der Ruprecht-Karls-Universität Heidelberg, Ärztekammer Schleswig-Holstein, Ärztekammer Niedersachsen, Land Berlin am Landesamt für Gesundheit und Soziales Berlin, Landesärztekammer Rheinland-Pfalz, Universität Ulm, Ärztekammer Nordrhein, Medizinische Fakultät der Universität Düsseldorf-Essen, Universität Regensburg, Ärztekammer Hamburg, Ärztekammer Westfalen-Lippe und der Medizinischen Fakultät der Westfälischen WilhelmsUniversität Münster and Medizinische Fakultät der Ludwig-Maximilians-Universität München-all Germany). The trial was performed in accordance with the Declaration of Helsinki. All patients provided written informed consent.
Consent to publish Not applicable.

Data availability Access to the anonymised dataset can be requested from the corresponding author.

Competing interests G.F. received a study grant by Merck and honoraria for lectures and advisory board meetings from Merck, Roche, Amgen, Lilly, SanofiAventis, Servier, Bayer, BMS and MSD. K.T. received honoraria for lectures and advisory board meetings from BMS, Celgene, Takeda, GSK, Servier and JanssenCilag. A.S. received research grants from Merck, BMS, Roche, Sanofi, Servier, German Cancer Aid and Federal Joint Committee, and honoraria for lectures and advisory board meetings by Merck, Roche, Amgen, Lilly, Sanofi-Aventis, Servier, Bayer, BMS, MSD and Sirtex. G.H. received honoraria for lectures and advisory board meetings by BMS, Sanofi-Aventis, Novartis, Roche and Boehringer Ingelheim. M.S. received honoraria for lectures from Merck and Amgen. SK received financial support for conduction of clinical trials by Merck, BMS; Roche, Celgene and Lilly and received honoraria for lectures and advisory board meetings from Merck, Roche, Amgen, Lilly, Sanofi-Aventis, Servier, Bayer, BMS, MSD and GSK. A.K.R.E. received honoraria for lectures and advisory board meetings or travel support for medical conference attendance by Merck, Roche, Amgen, Sanofi-Aventis, Servier, BMS and MSD. C.H.K. received a study grant and honoraria for lectures and advisory board meetings from Merck, Roche, Amgen, Lilly, Sanofi-Aventis, Servier, Bayer, BMS, MSD and Novartis. V.G. acted as an advisor for BMS, MSD, Merck Serono, AstraZeneca, Novartis, Pfizer, Ipsen, Cerulean, EUSA-Pharm., Roche and Nanobiotix and received compensation for lectures from BMS, MSD, Merck Serono, AstraZeneca, Novartis, Pfizer, Ipsen, Eisai, Roche, PharmaMar, Lilly, Janssen-Cliag and Exelexis. He received research grants from AstraZeneca, BMS, MSD, Pfizer and Ipsen. C.B. received honoraria for lectures and advisory board meetings from Lilly, Merck, Sanofi, Roche Mundipharma, Bayer, Hexal, GSO, BMS, Servier, Pfizer and AstraZeneca. A.K.R.Ä. received research support by Bayer and Merck Serono, study grants by Merck and BMS and honoraria for lectures and advisory board meetings, as well as reimbursement for study-related travels by Roche and Daiichi-Sankyo. All other authors declare no conflict of interest.

Funding information The trial was supported by the German Arbeitsgemeinschaft für Internistische Onkologie (AIO), CUP working group and by an unrestricted grant and provision of cetuximab by Merck-Serono $\mathrm{GmbH}$, Darmstadt, Germany, an affiliate of Merck KGaA, Darmstadt, Germany. Open Access funding enabled and organized by Projekt DEAL.

Publisher's note Springer Nature remains neutral with regard to jurisdictional claims in published maps and institutional affiliations.

\section{REFERENCES}

1. Rassy, E. \& Pavlidis, N. The currently declining incidence of cancer of unknown primary. Cancer Epidemiol. 61, 139-141 (2019).

2. Pavlidis, N. \& Pentheroudakis, G. Cancer of unknown primary site. Lancet. 379, 1428-1435 (2012).

3. Conway, A.-M., Mitchell, C., Kilgour, E., Brady, G., Dive, C. \& Cook, N. Molecular characterisation and liquid biomarkers in carcinoma of unknown primary (CUP): taking the 'U' out of 'CUP.'. Br. J. Cancer 120, 141-153 (2019).

4. Jones, W., Allardice, G., Scott, I., Oien, K., Brewster, D. \& Morrison, D. S. Cancers of unknown primary diagnosed during hospitalization: a population-based study. BMC Cancer 17, 85 (2017).

5. Varghese, A. M., Arora, A., Capanu, M., Camacho, N., Won, H. H., Zehir, A. et al. Clinical and molecular characterization of patients with cancer of unknown primary in the modern era. Ann. Oncol. 28, 3015-3021 (2017).

6. Hainsworth, J. D., Rubin, M. S., Spigel, D. R., Boccia, R. V., Raby, S., Quinn, R. et al. Molecular gene expression profiling to predict the tissue of origin and direct sitespecific therapy in patients with carcinoma of unknown primary site: A prospective trial of the Sarah cannon research institute. J. Clin. Oncol. 31, 217-223 (2013).

7. Briasoulis, E., Kalofonos, H., Bafaloukos, D., Samantas, E., Fountzilas, G., Xiros, N. et al. Carboplatin plus paclitaxel in unknown primary carcinoma: a Phase II Hellenic Cooperative Oncology Group Study. J. Clin. Oncol. 18, 3101-3107 (2000).

8. Greco, F. A. Cancer of unknown primary site: improved patient management with molecular and immunohistochemical diagnosis. Am. Soc. Clin. Oncol. Educ. B. 33, 175-181 (2013).

9. Huebner, G., Link, H., Kohne, C. H., Stahl, M., Kretzschmar, A., Steinbach, S. et al. Paclitaxel and carboplatin vs gemcitabine and vinorelbine in patients with 
adeno- or undifferentiated carcinoma of unknown primary: a randomised prospective phase II trial. Br. J. Cancer 100, 44-49 (2009).

10. Yoon, H. H., Foster, N. R., Meyers, J. P., Steen, P. D., Visscher, D. W., Pillai, R. et al. Gene expression profiling identifies responsive patients with cancer of unknown primary treated with carboplatin, paclitaxel, and everolimus: NCCTG N0871 (alliance). Ann. Oncol. 27, 339-344 (2016).

11. Hainsworth J. D., Daugaard G., Lesimple T., Hübner G., Greco F. A., Stahl M. J. et al. Paclitaxel/carboplatin with or without belinostat as empiric first-line treatment for patients with carcinoma of unknown primary site: a randomized, phase 2 trial. Cancer 121, 1654-1661 (2015).

12. Hainsworth, J. D., Spigel, D. R., Thompson, D. S., Murphy, P. B., Lane, C. M. Waterhouse, D. M. et al. Paclitaxel/carboplatin plus bevacizumab/erlotinib in the first-line treatment of patients with carcinoma of unknown primary site. Oncologist 14, 1189-1197 (2009).

13. Vermorken, J. B., Mesia, R., Rivera, F., Remenar, E., Kawecki, A., Rottey, S. et al. Platinum-based chemotherapy plus cetuximab in head and neck cancer. N. Engl. J. Med. 359, 1116-1127 (2008).

14. Bokemeyer, C., Bondarenko, I., Makhson, A., Hartmann, J. T., Aparicio, J., De Braud, F. et al. Fluorouracil, leucovorin, and oxaliplatin with and without cetuximab in the first-line treatment of metastatic colorectal cancer. J. Clin. Oncol. 27, 663-671 (2009).

15. Van Cutsem, E., Köhne, C.-H., Hitre, E., Zaluski, J., Chang Chien, C.-R., Makhson, A et al. Cetuximab and chemotherapy as initial treatment for metastatic colorectal cancer. N. Engl. J. Med. 360, 1408-1417 (2009).

16. Ciardiello, F. \& Tortora, G. EGFR Antagonists in cancer treatment. N. Engl. J. Med. 358, 1160-1174 (2008).

17. Pentheroudakis, G., Golfinopoulos, V. \& Pavlidis, N. Switching benchmarks in cancer of unknown primary: From autopsy to microarray. Eur. J. Cancer 43, 2026-2036 (2007).

18. Fizazi, K., Greco, F. A., Pavlidis, N., Daugaard, G., Oien, K. \& Pentheroudakis, G. Cancers of unknown primary site: ESMO Clinical Practice Guidelines for diagnosis, treatment and follow-up. Ann. Oncol. 26, v133-v138 (2015).

19. Therasse, P., Arbuck, S. G., Eisenhauer, E. A., Wanders, J., Kaplan, R. S., Rubinstein L. et al. New guidelines to evaluate the response to treatment in solid tumors. J. Natl. Cancer Inst. 92, 205-216 (2000).
20. Douillard, J.-Y., Oliner, K. S., Siena, S., Tabernero, J., Burkes, R., Barugel, M. et al Panitumumab-FOLFOX4 treatment and RAS mutations in colorectal cancer. $N$. Engl. J. Med. 369, 1023-1034 (2013).

21. Moran, S., Martínez-Cardús, A., Sayols, S., Musulén, E., Balañá, C., Estival-Gonzalez, A. et al. Epigenetic profiling to classify cancer of unknown primary: a multicentre, retrospective analysis. Lancet Oncol. 17, 1386-1395 (2016).

22. Hayashi, H., Kurata, T., Takiguchi, Y., Arai, M., Takeda, K., Akiyoshi, K. et al. Randomized Phase II trial comparing site-specific treatment based on gene expression profiling with carboplatin and paclitaxel for patients with cancer of unknown primary site. J. Clin. Oncol. 37, 570-579 (2019).

23. Fizazi K., Maillard A., Penel N. et al. A phase 3 trial of empiric chemotherapy with cisplatin and gemcitabine or systemic treatment tailored by molecular gene expression analysis in patients with carcinomas of an unknown primary (CUP) site (GEFCAPI 04). Present ESMO Congr 2019, Barcelona Spain; LBA15_PR.

24. Rassy, E., Bakouny, Z., Choueiri, T. K., Van Allen, E. M., Fizazi, K., Greco, F. A. et al. The role of site-specific therapy for cancers of unknown of primary: a metaanalysis. Eur. J. Cancer 127, 118-122 (2020).

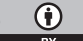

Open Access This article is licensed under a Creative Commons Attribution 4.0 International License, which permits use, sharing, adaptation, distribution and reproduction in any medium or format, as long as you give appropriate credit to the original author(s) and the source, provide a link to the Creative Commons license, and indicate if changes were made. The images or other third party material in this article are included in the article's Creative Commons license, unless indicated otherwise in a credit line to the material. If material is not included in the article's Creative Commons license and your intended use is not permitted by statutory regulation or exceeds the permitted use, you will need to obtain permission directly from the copyright holder. To view a copy of this license, visit http://creativecommons. org/licenses/by/4.0/.

(c) The Author(s) 2020 